\title{
Risk management of infectious disease using multidimensional Omics: Molecular diagnostic and personal care of tuberculosis
}

\author{
Amir Buzimkic, Diane E Heck and Hong Duck Kim * \\ Department of Public Health, School of Health Sciences and Practice, New York Medical College, Valhalla NY.
}

GSC Biological and Pharmaceutical Sciences, 2021, 15(01), 001-004

Publication history: Received on 04 February 2021; revised on 12 March 2021; accepted on 15 March 2021

Article DOI: https://doi.org/10.30574/gscbps.2021.15.1.0071

\begin{abstract}
Tuberculosis (TB) is one of the top 10 leading causes of death worldwide responsible for over 1.5 million deaths annually. It is caused by hazardous biological pathogen (i.e., Mycobacterium tuberculosis (MTB)) with single infectious agent, surpassing even HIV/AIDS. Roughly one-quarter of the world's population has latent TB, meaning that people have been infected by tuberculosis bacteria but have not yet developed the disease. Patients with active tuberculosis on average infect five to fifteen other people via airborne droplets. Once infected, people with HIV are 19 times more likely to develop active tuberculosis which has almost $100 \%$ mortality for this group, if not treated properly. Comparatively, $45 \%$ of HIV negative people will die if they develop active tuberculosis and are not adequately medicated. This is concerning since $95 \%$ of cases and deaths are in developing countries, where treatments and diagnosis may not be timely. Additionally, current detection methods do not distinguish active tuberculosis from a cleared or latent infection while microbiological culture of mycobacteria is slow. However, medical discoveries and newly developed technologies allowed for unification of disciplines incorporating omics into everyday biological research. The goal of this short review is to demonstrate ways in which field of multidimensional Omics could contributed to the advanced detection of infectious disease by improving accuracy and quality of patient care by implementing molecular based detection of pathogen (i.e., antigenicity and metabolomics tools) as well as personal care with follow-up monitoring care (i.e., immunogenicity and vaccinomics tools) in the diagnosis, treatment, and prevention of tuberculosis.
\end{abstract}

Keywords: Multidimensional Omics; Infectious disease; Whole genome sequencing; Tuberculin Skin Test (TST); Interferon Gamma Release Assay (IGRA); Mycobacterium tuberculosis.

\section{Introduction}

Tuberculosis (TB) has been a factor of environmental stress on our health since the beginning of human existence. The earliest discovery of TB in humans dates back over 9000 years. Archaeologists discovered TB in the remains of a mother and child which were buried together in small Mediterranean town. Throughout the history the diseases caried names such as "phthisis", "schachepheth", "the white plague", and "consumption". Earliest attempts of treatment, mostly including warmth, good food, and rest, were not very successful. Early Europeans even resorted to remedies such as the "royal touch", where they reasoned that a touch by English and French monarch would cure the disease [4,5]. In 1882, it was discovered that tuberculosis is caused by bacterium called Mycobacterium tuberculosis (MTB). Patients were starting to get isolated into sanatoriums for treatments with fresh air, good food and on occasion, even surgery. It was not until the 1940s, with the discovery of antibiotics, that treatment for TB was revolutionized [5]. Interestingly, not everyone infected with Mycobacterium tuberculosis shows indications of the dieses and becomes ill. Some people can carry the bacteria in their body for years without developing symptoms or being infective. As a result, TB is classified into two conditions: latent TB infection (LTBI) and TB disease. Both conditions are generally detected by one of the two

\footnotetext{
${ }^{*}$ Corresponding author: Hong Duck Kim

Department of Public Health, School of Health Sciences and Practice, New York Medical College, Valhalla NY.
} 
tests: Tuberculin Skin Test (TST) or Interferon Gamma Release Assay (IGRA). However, these tests do not discriminate between LTBI and active TB disease and further screening is necessary for positive patients $[5,18,21]$.

Recent advances in the fields of omics, which consist of two modules: genomics, transcriptomics and proteomics as core omics and metabolomics, immunomics and vaccinomics as advanced Omics, offers opportunities to empower systematic tools for TB diagnostics, treatment, and prevention. By examining integrated analysis of these studies, we can identify unified reaction to the infection and advance global eradication efforts.

\section{Application of Omics in Tuberculosis Diagnostics}

The gold standard in TB and latent TB infection (LTBI) testing is the Tuberculin Skin Test (TST), also known as the Mantoux test, named after Charles Mantoux (1877-1947) who established this diagnostic criterion. The test is performed by intradermally injecting purified protein derivative (PPD) reagent into the forearm. In composition, PPD is $92.9 \%$ protein, $5.9 \%$ polysaccharide, and $1.2 \%$ nucleic acid. The delayed-type hypersensitivity (DTH) response is monitored 48 to 72 hours post-injection by measuring the diameter of induration. The diameter of induration and exposure status determine the result. The main concern with the TST is the high-level of false positive results, triggered by the inability of the test to distinguish between TB disease, LTBI, and exposure to Bacillus Calmett-Guérin (BCG) [22]. Similarly, Interferon Gamma Release Assay (IGRA) tests which detect the presence of MTB via in vitro release of interferon-gamma upon stimulation from previously sensitized $\mathrm{T}$ cells, cannot differentiate between TB disease and LTBI [6] (Chang \& Leung, 2010). However, IGRA can prevent false positive results caused by the BCG vaccination [10].

In term of microbiological diagnosis, microscopy is the most used approach. Nevertheless, it heavily relies on abundance of MTB in the sample and skillset of the operator. Cultures can increase sensitivity, but they have a slow generation time and can take weeks to identify MTB $[1,11]$.

Recent developments in genomics provide the opportunity to improve TB diagnostics by enhancing bacterial detection. Whole genome sequencing (WGS) has been used to differentiate between relapse and re-infection cases, find mutations which enable drug resistance, and track transmission events. Furthermore, WGS can inform on antibiotic susceptibility of MTB via public databases of given drug resistance $[14,15]$

Another advantage of WGS is its ability to reduce the wait period by identifying the organism within 72 hours. Furthermore, MTB genome can be sequenced directly from uncultured sputum sample which is beneficial for clinically relevant time frame. One downfall of this method can be seen by examining culture-negative sputum samples (collected from treated patients) which indicate that DNA-based methods cannot differentiate active disease from cleared infections [8].

Several dozen studies assessing human transcriptional response to TB have been published in the last decade, however, no diagnostic test utilizing this technology exists. Additionally, blood transcriptomics yead little result since profiling at the site of disease may produce significant results that may not be apparent in blood [16].

Lastly, proteomic and metabolomic approach to TB biomarker discovery faces issues of data availability, validation, standardization, and reproducibility. The overlap between independent studies and lack of cross validation indicates that optimal metabolic biosignature have not been noted indicating the need for further studies [2,8].

\section{Application of Omics in Tuberculosis Treatment}

TB disease is currently treated by one, or combination of few drugs which are taken in duration of 6 to 9 months. There are currently 10 drugs approved by the U.S. Food and Drug Administration (FDA) for treatment of TB. The most used regiments are isoniazid (INH), rifampin (RIF), ethambutol (EMB), and pyrazinamide (PZA) [19]. However, the challenge of antimicrobial drug resistance (AMR) is on the rise, with AMR associated with $3.4 \%$ of new TB cases worldwide and up to $50 \%$ of previously treated cases in some regions [19].

Comprehensive WGS of MTB has revealed a core genome and mapped accumulation of single nucleotide polymorphisms (SNPs) in protein coding genes. Furthermore, due to comparative genomics and by examining the presence or absence of target protein coding, toxicity and drug specificity can now be anticipated. This has provided us with framework for potential medication targets. Moreover, manipulation of gene functions has shed light on pathways essential to MTB in various microenvironments emphasizing targets for drug breakthroughs [7]. 
In previous study focus on the transcriptional perspective, exposure to MTB to antibiotics has improved our understanding of many medications presently in use, as well as predicting mode of action and the targets for new drugs. For instance, Wilson et al.(1999) reported that one of genomic tool could benefit to explore changes in gene expression and demonstrate that drug treatment with INH caused a cluster of 5 genes encoding type II fatty acid synthase enzymes (fabD-acpM-kasA-kasB-accD6) to be upregulated by DNA microarrays [17]. Presently, there are promising results indicating new ways to facilitate drug evaluation and validation process using transcriptomics platform which has been utilized to examine the mode of action of drugs in phase I/II studies [7].

Proteomics contribution in deconvolution of MTB responses to chemical exposure has provided an insight into mechanisms of drug action and antimicrobial drug resistance (AMR). Noteworthy, Vijayaraj et al. (2019) reported that multidrug resistance gene in TB (MDR-TB) could affect upon molecular behavioral either of UDP galactopyranose and GDP-L-galactose which may alter binding and inhibition activity coupling with key of multidrug resistance regulator such as transcriptional regulator protein, WhiB6, followed by proteomic and gene expression profile complimented approach [20]. Similarly, metabolomics and lipidomics demonstrated its relevancy in studies which explain mode of action of new compounds. A recent study led by Sharma et al. demonstrated interaction between genomic and functionality in which impact of the natural antimycobacterial compound vanillin in M. smegmatis was mapped, found that vanillin changes the composition of fatty acids causing disruption of cell membrane homeostasis [14].

\section{Application of Omics in Tuberculosis Prevention}

Current TB prevention methods include identification of LTBI patients and their treatment with above mentioned regiment of antibiotics. Additionally, BCG vaccine is used in some countries as prevention against TB meningitis in children, against which is has 70-80\% effectiveness. There are still debates on effectiveness of BCG in prevention of, more common, respiratory TB disease in adults [19]. Due to the advent of the genomics era, it is possible to amend to verify the issue of an established systemic approach combined with a health inventory system and vaccination center. To date, Omics technologies used in inoculation research are often described by the term "vaccinomics." Such technologies merged with bioinformatics form a powerful approach used for vaccine target identification. Furthermore, it is important to weigh of prevention to uncertain pathogenicity use omics platform like genomics-based reverse vaccinology which predicts the immunization protein candidates vie analysis of genome sequences $[9,13]$.

The way we are focused on genes of interest or potential genes which could be involved in microbial pathogenesis was revolutionized by high-throughput transcriptomics analysis of gene expression by using DNA microarray. This resulted in creation of significant amounts of gene expression data produced by assay systems ability to measure the expression pattern of thousands of genes in parallel. These microarrays can be hybridized with complemental DNA (from microorganisms' messenger RNA) which can be used for whole transcriptome shotgun sequencing to get information about a sample's RNA contents. Ultimately, it is possible and important to note genes expressed during the infection since they are the most likely protective immunizations targets [9].

\section{Conclusion}

Multidimensional Omics play a key role in the field of disease prevention and prediction including clinical diagnosis, treatment, and prevention of TB in the future. These molecular platform-based technologies are multiplied in strengths when used in conjunction and can help understand bacterial metabolic pathogenicity. Further work will require a systemic approach involving development and maintenance of big data management such as data repositories, bioinformatics tools as well as integration and visualization platforms readily accessible to the research community. Significant reductions of cost due to era of genomic, for example DNA sequence and gene mapping tool kits, in cost of omics technology alongside increased accessibility to data will result in unparalleled advances in diagnostic, clinical and preventive capabilities expanded more challenge areas in the pulmonary and infectious disease such as Covid-19 pandemic.

\section{Compliance with ethical standards}

\section{Acknowledgments}

This work was in part supported by the Student fund in School of Health Science and Practice and Institute of Public Health, New York Medical College. 


\section{Disclosure of conflict of interest}

These authors declare no conflict of financial interest.

\section{References}

[1] Boehme CC, Saacks S, O'Brien RJ. The Changing Landscape of Diagnostic Services for Tuberculosis. Seminars in Respiratory and Critical Care Medicine. 2013; 34(01): 17.

[2] Cai Y, Yang Q, Tang Y, Zhang M, Liu H, Zhang G, Deng Q, Huang J, Gao Z, Zhou B, Feng CG, Chen X. Increased Complement C1q Level Marks Active Disease in Human Tuberculosis. PloS One. 2014; 9(3): e92340.

[3] Centers for Disease Control and Prevention. History of the World TB Day. 2019.

[4] Centers for Disease Control and Prevention. Treatment for TB Disease. 2019.

[5] Centers for Disease Control and Prevention. Testing for Tuberculosis. 2020.

[6] Chang KC, Leung CC. Systematic review of interferon-gamma release assays in tuberculosis: focus on likelihood ratios. Thorax. 2010; 65(3): 271-276.

[7] Goff A, Cantillon D, Muraro Wildner L, Waddell SJ. Multi-Omics Technologies Applied to Tuberculosis Drug Discovery. Applied Sciences. 2020; 10(13): 4629.

[8] Haas CT, Roe JK, Pollara G, Mehta M, Noursadeghi M. Diagnostic 'omics' for active tuberculosis. BMC Medicine. 2016; 14(1): 37.

[9] He Y. Omics-Based Systems Vaccinology for Vaccine Target Identification. Drug Development Research. 2012; 73(8): 559-568.

[10] Meissner HC. Options for diagnosing asymptomatic (latent) tuberculosis infection. American Academy of Pediatrics. 2018.

[11] Molicotti P, Bua A, Zanetti S. Cost-effectiveness in the diagnosis of tuberculosis: choices in developing countries. The Journal of Infection in Developing Countries. 2014; 8(01): 24.

[12] National Health Institute. BCG tuberculosis (TB) vaccine overview. nhs.uk. 2019.

[13] Nazir Z, Afridi SG, Shah M, Shams S, Khan A. Reverse vaccinology and subtractive genomics-based putative vaccine targets identification for Burkholderia pseudomallei Bp1651. Microbial Pathogenesis. 2018; 125: 219229.

[14] Sharma S, Hameed S, Fatima Z. Lipidomic insights to understand membrane dynamics in response to vanillin in Mycobacterium smegmatis. International Microbiology: The Official Journal of the Spanish Society for Microbiology. 2020; 23(2): 263-276.

[15] Walker TM, Lalor MK, Broda A, Ortega LS, Morgan M, Parker L, Churchill S, Bennett K, Golubchik T, Giess AP, Elias C, Jeffery KJ, Bowler I, Laurenson IF, Barrett A, Drobniewski F, McCarthy ND, Anderson LF, Abubakar I, and Conlon CP. Assessment of Mycobacterium tuberculosis transmission in Oxfordshire, UK, 2007-12, with whole pathogen genome sequences: an observational study. 2014; 2(4): 285-292.

[16] Walter ND, Miller MA, Vasquez J, Weiner M, Chapman A, Engle M, Higgins M, Quinones AM, Rosselli V, Canono E, Yoon C, Cattamanchi A, Davis JL, Phang T, Stearman RS, Datta G, Garcia BJ, Daley CL, Strong M, Geraci MW. Blood Transcriptional Biomarkers for Active Tuberculosis among Patients in the United States: a Case-Control Study with Systematic Cross-Classifier Evaluation. Journal of Clinical Microbiology. 2016; 54(2): 274-282.

[17] Wilson M, DeRisi J, Kristensen HH, Imboden P, Rane S, Brown PO, Schoolnik GK. Exploring drug-induced alterations in gene expression in Mycobacterium tuberculosis by microarray hybridization. Proceedings of the National Academy of Sciences of the United States of America. 1999; 96(22): 12833-12838.

[18] World Health Organization. The top 10 causes of death. 2018.

[19] World Health Organization. Global tuberculosis report 2019. WHO. 2019.

[20] Vijayaraj M, Abhinand PA, Ragunath PK. Virtual screening of a MDR-TB WhiB6 target identified by gene expression profiling Bioinformation. 2019; 15(8): 557-567.

[21] World Health Organization. Tuberculosis (TB). 2020.

[22] Yang H, Kruh-Garcia NA, Dobos KM. Purified Protein Derivatives of Tuberculin - Past, Present, and Future. FEMS Immunology and Medical Microbiology. 2012; 66(3): 273-280. 